\title{
Importance of high-performing teams in the cardiovascular intensive care unit
}

Lauren R. Kennedy-Metz, PhD, ${ }^{\mathrm{a}, \mathrm{b}}$ Atilio Barbeito, MD, MPH, ${ }^{\mathrm{c}, \mathrm{d}}$ Roger D. Dias, MD, MBA, PhD, ${ }^{\mathrm{e}}$ and Marco A. Zenati, MD ${ }^{\mathrm{a}, \mathrm{b}}$

The importance of teamwork in health care delivery and patient safety is increasingly being recognized ${ }^{1}$ and has benefitted substantially by adopting a human factors' perspective and approach. The scientific field of human factors can be defined in many ways, but the commonality across definitions and applications is the focus on those components within an interactive system related to human functionality and fallibility. By recognizing human-centric and human-driven strengths and flaws in a complex system, we may be able to better understand their root causes to maintain performance-enhancing behaviors and improve or support error-generating behaviors. As systems increase in complexity, consequences of suboptimal performance become more severe, demands become excessive and timesensitive, and human lives are at stake, relying on teams represents a natural solution to enhance perspective, decision-making, and global knowledge beyond the individual level. ${ }^{2}$

In a system as complex as health care, optimal teamwork requires collaboration within and across organizational, disciplinary, technical, and cultural boundaries. Although recent literature has indicated a substantial role of cognitive errors in the occurrence of adverse events in health care, teamwork and communication combined were identified as human performance deficiencies contributing to adverse events in more than $17 \%$ of cases. ${ }^{3}$ According to Salas and colleagues' teamwork model, ${ }^{4}$ effective teamwork is based on the quality of team leadership, mutual performance monitoring, back-up behavior, adaptability, and a team orientation at its core. In addition, mutual trust, closed-loop communication, and shared mental models underlie and coordinate the 5 core dimensions in this model. Of these features, the shared mental

From the Departments of a Surgery, and ${ }^{\mathrm{e} E m e r g e n c y ~ M e d i c i n e, ~ H a r v a r d ~ M e d i c a l ~}$ School, Boston, Mass; ${ }^{c}$ Anesthesiology Service, Durham VA Health Care System, Durham, NC; ${ }^{\mathrm{d}}$ Department of Anesthesiology, Duke University, Durham, NC; and ${ }^{\mathrm{b}}$ Division of Cardiac Surgery, VA Boston Healthcare System, Boston, Mass.

This work was supported by the National Heart, Lung, and Blood Institute, National Institutes of Health (R01HL126896, Principal Investigator M.A.Z.). The content is solely the responsibility of the authors and does not necessarily represent the official views of the National Institutes of Health.

Received for publication Dec 15, 2020; revisions received Feb 22, 2021; accepted for publication Feb 23, 2021; available ahead of print March 31, 2021.

Address for reprints: Marco A. Zenati, MD, Division of Cardiac Surgery, Veterans Affairs Boston Healthcare System, 1400 VFW Parkway, Boston, MA 02132 (E-mail: marco_zenati@hms.harvard.edu).

J Thorac Cardiovasc Surg 2022;163:1096-104

0022-5223/\$0.00

Published by Elsevier Inc. on behalf of The American Association for Thoracic Surgery

https://doi.org/10.1016/j.jtcvs.2021.02.098

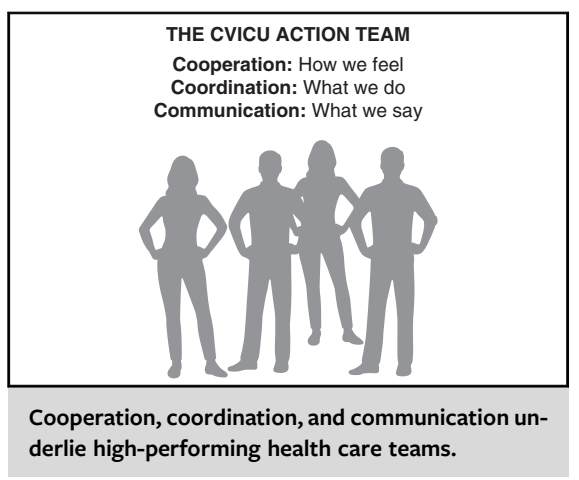

CENTRAL MESSAGE

The CVICU team is a complex

action team providing care to

high-acuity patients in a chal-

lenging environment. Evidence-

based teamwork interventions

are critical to deliver high-quality

care in the CVICU.

See Commentaries on pages 1105 and 1106.

model serves as the cornerstone for effective teamwork in health care ${ }^{5}$ and has been ranked as one of the most essential teamwork factors in cardiac surgery in particular. ${ }^{6}$

A shared mental model refers to the shared cognition in a team as a collectivity ${ }^{7}$ and may be described as the extent to which individuals possess a similar cognitive representation of some situation. Effective shared mental models produce mutual awareness, with which team members can reason about not only their own situation but also the status of team members in the pursuit of joint goals. Shared mental models are proposed to explain certain coordinated team behaviors. Common knowledge (beliefs) of the domain problem under concern, communication protocols to use, and assumptions to take (eg, sincerity, communication reliability) establishes a common basis for agents to understand and respond to each other's behaviors. A team structure may specify such information as team membership, subteam relations, predetermined leader, roles each member can play, capability requirements, and so forth. To have a shared team structure enables an individual to develop a higher-level 
abstraction about the capabilities, expertise, and responsibilities of other team members.

Among high-performing medical teams, interdisciplinary teamwork has been associated with improved patient outcomes in emergency medicine, ${ }^{8}$ critical care medicine, ${ }^{9}$ and internal medicine ${ }^{10}$ through various settings. Effective teamwork skills have also been documented as significant predictors for patient outcomes, demonstrated through medical simulation involving participants across health professions. ${ }^{11,12}$ In surgical departments, poor communication and information sharing, both critical teamwork behaviors, have been associated with higher occurrences of complications and death. ${ }^{13}$ Meanwhile, discrepancies exist between perceived teamwork levels in operative settings, ${ }^{14}$ complicating the ability to accurately measure and improve teamwork.

Additional barriers to effective teamwork in the operating room (OR) environment exist on individual, environmental, institutional levels, and cultural levels. ${ }^{15}$ Examples range from how an individual copes with cognitive demands, to environmental barriers associated with uneven lighting and excessive noise, to institutional burdens associated with teaching requirements in academic hospital settings, to the cultural implications of a deeply rooted hierarchical system with an inherent imbalance among power dynamics. Highly functioning teams are tasked with overcoming these and additional barriers under oftentimes adverse and time-sensitive conditions during a patient's hospital stay. Additionally, patient care and interdisciplinary team interaction continue beyond the OR, where effective and dynamic teamwork is critical in both the handover process and the patient's intensive care stay.

In surgery, recent initiatives have been adopted in the United States and worldwide, ${ }^{16-18}$ aiming to train surgical providers, from novices to senior levels, on important nontechnical skills, such as situational awareness, leadership, decision-making, communication, and teamwork. In fact, in 2016, the American College of Surgeons adopted and recommended nontechnical skills training beginning during undergraduate medical education and continuing through surgical residency and postgraduate training as a requirement of ongoing Maintenance of Certification. ${ }^{19}$ Furthermore, the Non-Technical Skills for Surgeons framework has implemented and validated to the US surgical context as a team-training platform. ${ }^{18}$

\section{TEAMWORK IN THE CARDIOVASCULAR INTENSIVE CARE UNIT}

The cardiovascular intensive care unit (CVICU), is a complex socio-technical system where critical care is delivered with the support of sophisticated technology to treat and prevent complications arising from advanced postsurgical care: The ultimate success of the delivery of care rests on the multidisciplinary team of clinical providers. ${ }^{20}$
The CVICU team may be described as an action team. Action teams are "interdisciplinary teams that often work under complex, dynamic, and time-pressured conditions to accomplish critical patient care tasks." ${ }^{21}$ Action teams differ from regular teams in that they are highly specialized, have a dynamic membership structure, incorporate different professional cultures, work together for short periods, and have to improvise and coordinate their actions in intense, unpredictable situations. ${ }^{22}$ The typical CVICU team is led by a physician specialized in intensive care medicine and is composed of bedside nurses, several respiratory therapists and physical therapists, a clinical pharmacist, a nutritionist, and occasionally other team members such as a psychologist and a chaplain. Given that the cardiothoracic critical care patient population is increasingly older and sicker, and surgical procedures are also growing in complexity, CVICU intensivists leading the team require a unique combination of skills that include basic critical care training plus additional skills and familiarity with cardiothoracic surgery and perioperative care principles. Recognizing this need, other surgical and medical societies have advocated for a specific training in cardiothoracic critical care and some critical care programs are offering combined critical care fellowship pathways with an emphasis in cardiothoracic care. ${ }^{23-25}$ Family members are also considered part of the team in most modern CVICUs. ${ }^{26}$ Each of these disciplines brings a unique set of perspectives, vocabulary, and skills, which typically add value to the care provided by the CVICU team.

Unfortunately, these differences also may lead to conflict and ineffective interactions among the team members. Unresolved conflict may further hinder communication and may perpetuate or accentuate poor team performance. ${ }^{27}$ Another characteristic of the CVICU team is the existence of hierarchies and power differences among the team members, which may inhibit information exchange, especially during rounds where most of the members are present and have the opportunity to speak up, and where shared goals are established or maintained. ${ }^{28}$

In addition to high patient acuity and complex team dynamics, CVICU teams perform under uniquely challenging physical and emotional constraints that may affect team performance. ${ }^{28}$ The challenging environment of constant alarms, uneven lighting, space limitations and improperly placed equipment typical of the CVICU all affect the performance of the team. ${ }^{29}$ Furthermore, the emotional distress brought on by stressors such as high workload, high-stakes situations, and frequent experiences of death and grieving can reduce team performance. ${ }^{30}$

\section{CARDIOVASCULAR INTENSIVE CARE UNIT HANDOVER AND TEAMWORK}

At the interface of perioperative and postoperative care, the patient is transitioned from the OR to the intensive 
care unit (ICU) teams during the "handover." ${ }^{31}$ Patient handover is fraught with potential risk for preventable technical and communication errors ${ }^{32}$; human-derived flow disruptions $^{33}$ are also common. Patients transitioning to the CVICU from cardiac surgery are particularly vulnerable to the negative outcomes associated with poor handovers. ${ }^{1}$ Previous work has suggested that up to $28 \%$ of surgical adverse events were documented during transitions of care, including the handover process. ${ }^{34}$ Further, during the postoperative handover in particular, adverse events are associated with poorly executed handovers. ${ }^{31}$

To adequately address and mitigate preventable errors during the handover, clinicians and patient safety experts have advocated for a human-centric approach. ${ }^{35}$ With appropriately designed interventions targeting teamwork and communication, it is possible to improve efficiency and effectiveness of the handover process. ${ }^{31}$ In summary, the CVICU team is an action team that provides care to a high-acuity patient population, exhibits complex team composition and dynamics, and operates in an emotionally and physically adverse environment. Activities targeted at improving teamwork are paramount to delivering highquality care in the CVICU.

\section{IMPROVING CARDIOVASCULAR INTENSIVE CARE UNIT TEAMWORK}

Despite the known necessity for effective teamwork, the health care domain has surprisingly underinvested in structured and evidence-based practices for managing teams and coordinating care. ${ }^{36}$ But given the strength of observed improvements in ICU teamwork and their association with improved patient outcomes, ${ }^{37-39}$ experts acknowledge the need to enhance teamwork, especially during the perioperative handover. ${ }^{40}$ Evidence suggests that teamwork in health care is a trainable skill. ${ }^{41}$ To improve teamwork dynamics, the first step is to measure teamwork components and identify barriers to effective teamwork. Tools used to measure teamwork in health care have largely relied on self-report or observational approaches. $^{42}$ A more objective approach is required to compensate for the shortcomings associated with these approaches. ${ }^{43}$ In addition to being more cost-effective and less biased, objective approaches incorporating sensor technology (eg, radio frequency identification tags, infrared sensors, audio/video recording devices, computer vision, and accelerometers) are capable of objectively capturing and evaluating teamwork behaviors (eg, behavior, speech analysis, team centrality, device, workplace locations) in an automated way. ${ }^{44}$

Barriers to interdisciplinary teamwork must then be identified and measured, preferably using multiple modalities, to target appropriate changes to introduce. Common obstacles facing ICU teamwork that have been identified previously include educational, psychological, and organizational/cultural barriers. ${ }^{15}$ In particular, factors such as the number of providers involved in the handover process, frequent trainee rotation schedules, and high staff turnover contribute to these barriers on many levels.

Tangible and accessible approaches to overcoming these barriers and promoting an environment of diligence may incorporate cognitive engineering approaches previously identified in cardiothoracic surgery, ${ }^{45}$ which are readily adaptable to the CVICU setting. In this context, the use of cognitive aids and emphasis on the "sterile cockpit" 46 have a high potential to influence behaviors and outcomes. Introducing a straightforward but standardized handover form prepared by the anesthesiologist to document the details of the procedure, patient requirements (eg, mechanical ventilation), team members involved, any complexities associated with the patient's course, and current lines and infusions functions to update the ICU nurse and has been shown to increase overall satisfaction with the handover process. ${ }^{35}$

A systematic review of handover characteristics concluded that the introduction of a standardized handover document was associated with significant improvements in the majority of studies. ${ }^{47}$ This finding has since been corroborated, in addition to the observed increase in confidence and reduction in perceived near-miss events, in a controlled trial evaluating the effectiveness of a structured handover tool in comparison with traditional methods of conducting the OR-ICU handover. ${ }^{48}$

In cardiac surgery in particular, the introduction of a standardized OR-CVICU handover protocol involving a multidisciplinary team demonstrated a significant reduction in technical errors and omissions of verbal handover information. $^{49}$ A more recent interventional study analyzing the transition from OR to CVICU additionally introduced a sterile cockpit time-out and observed fewer handover interruptions and more frequent postoperative patient care planning, without a substantial increase in handover duration. Participants also reported improvements in self-perceived teamwork, content received, and patient care planning. $^{50}$

Additional cognitive aids, such as wall-mounted posters, may facilitate the handover process further by reminding providers of critical elements and providing additional structure (eg, Figure 1). Encouraging nursing staff to play an active role in listening, asking questions, and taking notes while the entire team is present for the duration of the handover ensures that team members are actively updating their mental models to drive optimal teamwork. Given the vulnerability of communication processes at transition points such as the handover, a focus on enhancing the quality and frequency of communication is strongly recommended as a method of improving the optimal teamwork required to produce an effective patient transition to the CVICU. 


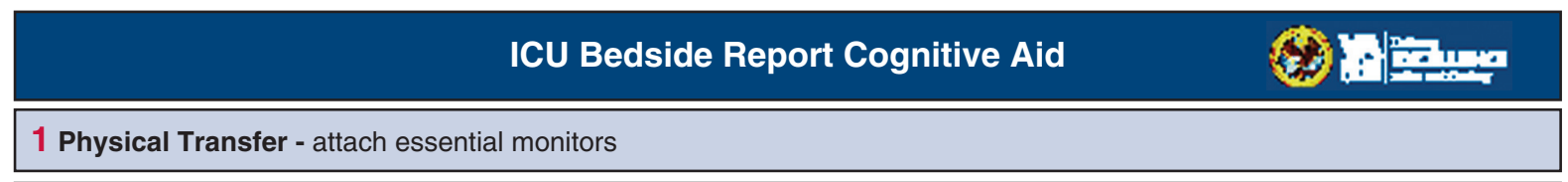

2 Information Transfer - at the foot of the bed, no interruptions, all members present

\begin{tabular}{|l|l|}
\hline Surgery & $\square$ Identify patient \\
& $\square$ Past medical history \\
& $\square$ Diagnosis - surgical procedure - intraoperative events \\
& $\square$ Surgical drains \\
& $\square$ Call back triggers \\
& $\square$ Potential complications for this procedure \\
& $\square$ Surgical coverage for the next 24 hours \\
\hline
\end{tabular}

\begin{tabular}{|ll|}
\hline Anesthesiology & $\square$ Allergies \\
& $\square$ Anesthetic details (drugs, doses) \\
& $\square$ Airway \\
& $\square$ I\&Os - blood products \\
& $\square$ Latest lab results and actions taken \\
& $\square$ Intraoperative Events \\
& $\square$ Antibiotics \\
& $\square$ Baseline cognitive and functional status
\end{tabular}

\begin{tabular}{|ll|}
\hline Physical Survey & $\square$ ET tube position $\quad \square$ Intravenouscatheter $\quad \square$ Surgical drains \\
& $\square$ Infusion pumps $\quad \square$ Pacemaker \\
\hline
\end{tabular}

\begin{tabular}{|ll|}
\hline Primary RN & $\square$ Any family available? \\
& $\square$ Have they been updated? \\
\hline
\end{tabular}

\section{Transfer of Care $\square$ Questions?}

口 OR to ICU Handover Redesign DIY Toolkit is produced by the Durham Patient Safety Center of Inquiry and VA National Center for Patient safety $\square$

FIGURE 1. An example of an OR-ICU handover cognitive aid currently used in one author's hospital (A.B.). ${ }^{35}$ This bedside poster to guide the handover process is included among other cognitive aids in a tool kit developed as part of a Veterans Affairs National Center for Patient Safety grant. ICU, Intensive care unit; I\&Os, intake and output.

To effectively integrate cultural changes in an environment such as the health care system, psychological safety must be established and fostered. Psychological safety in health care environments refers to willingness to take interpersonal risks at work, whether to admit an error, ask a question, seek help, or simply say "I don't know." Although it seems intuitive that a behavioral style of health care teams that encourages criticism-free team communication should result in better clinical outcomes, Edmondson ${ }^{51}$ found that hospital units characterized by high-quality relationships, supportive and available leadership, and expectations of excellence somewhat surprisingly had higher error rates. The hypothesis to explain this paradox is that highfunctioning teams were not committing more errors; they were just more able to disclose, discuss, and learn from them. It is important to realize that only a small fraction of errors lead to patient harm (eg, those errors that lead to an "error cycle"), and most of them are related to being an intrinsically fallible human being. To consider errors as "treasures" means to take full advantage of the learning opportunity provided by disclosure of errors or near misses in environments that are psychologically safe. Notably, recent work has demonstrated the modifiable nature of psychological safety through a systematic team training framework, providing evidence of trends toward improvements in teamwork, communication, psychological safety, and patient outcomes. ${ }^{52}$

Simulation-based training is an effective method to improve not only technical skills but also teamwork in a variety of health care settings, ${ }^{53}$ including the CVICU. ${ }^{54-57}$ It is already known that high-fidelity simulation can provoke similar levels of stress in clinicians when compared with real clinical care during medical emergencies, corroborating the psychological fidelity of the simulation environment. ${ }^{58}$ Additionally, previous studies have highlighted the role of simulation on improving adverse event reporting 
TABLE 1. Actionable advice for developing high-functioning teams

\begin{tabular}{|c|c|}
\hline Action & Examples from the literature \\
\hline Foster a culture of psychological safety & $\begin{array}{l}\text { Edmondson AC. Learning from mistakes is easier said than done: group and organizational influences } \\
\text { on the detection and correction of human error. J Appl Behav Sci. } 2004 ; 40: 66-90 .^{51} \\
\text { Ridley CH, et al. Building a collaborative culture: focus on psychological safety and error reporting. } \\
\text { Ann Thorac Surg. } 2021 ; 111: 683-9 .^{52}\end{array}$ \\
\hline Measure teamwork & $\begin{array}{l}\text { Rosen MA, et al. How can team performance be measured, assessed, and diagnosed? In: Salas E, Frush } \\
\text { K, eds. Improving Patient Safety Through Teamwork and Team Training. Oxford, UK: Oxford } \\
\text { University Press; 2012:59-79. } \\
\text { Jeffcott SA, et al. Measuring team performance in healthcare: review of research and implications for } \\
\text { patient safety. J Crit Care. } 2008 ; 23: 188-96 .{ }^{43}\end{array}$ \\
\hline Standardize processes & $\begin{array}{l}\text { Joy BF, et al. Standardized multidisciplinary protocol improves handover of cardiac surgery patients to } \\
\text { the intensive care unit. Pediatr Crit Care Med. } 2011 ; 12: 304-8 .^{49} \\
\text { Gleicher Y, et al. Improving cardiac operating room to intensive care unit handover using a } \\
\text { standardised handover process. BMJ Open Qual. } 2017 ; 6: \mathrm{e} 000076 .^{50}\end{array}$ \\
\hline Brief and debrief & $\begin{array}{l}\text { Arriaga AF, et al. Real-time debriefing after critical events: exploring the gap between principle and } \\
\text { reality. Anesthesiol Clin. 2020;38:801-20. }\end{array}$ \\
\hline Use cognitive aids and checklists & $\begin{array}{l}\text { Arriaga AF, et al. Simulation-based trial of surgical-crisis checklists. N Engl J Med. 2013;368:246- } \\
53 .^{70}\end{array}$ \\
\hline Engage in team building activities & $\begin{array}{l}\text { Rasmussen MB, et al. Social ties influence teamwork when managing clinical emergencies. BMC Med } \\
\text { Educ. 2020;20:1-7. }\end{array}$ \\
\hline Learn as a team (eg, M\&M conferences) & $\begin{array}{l}\text { Eddy K, et al. Health professionals' experience of teamwork education in acute hospital settings: a } \\
\text { systematic review of qualitative literature. JBI Database Syst Rev Implement Rep. 2016;14:96- } \\
137 .^{72}\end{array}$ \\
\hline Practice as a team (eg, in situ simulations) & $\begin{array}{l}\text { Lutgendorf MA, et al. Multidisciplinary in situ simulation-based training as a postpartum hemorrhage } \\
\text { quality improvement project. Mil Med. } 2017 ; 182: \text { e1762-6. } \\
\text { Steinemann S, et al. In situ, multidisciplinary, simulation-based teamwork training improves early } \\
\text { trauma care. } J \text { Surg Educ. } 2011 ; 68: 472-7 .^{74}\end{array}$ \\
\hline
\end{tabular}

$M \& M$, Morbidity and mortality.

and mitigating failure-to-rescue (FTR) situations in acute settings. ${ }^{59-61}$ Existing validated frameworks, such as the TeamSTEPPS Training Curriculum, ${ }^{62}$ can be used to design high-fidelity scenarios and implement interprofessional simulations focused on learning objectives related to teamwork domains tailored to the CVICU context. Furthermore, behavioral marker instruments, such as the Team Emergency Assessment Measure, ${ }^{63}$ Non-Technical Skills for Surgeons, ${ }^{64}$ and TeamSTEPSS Team Performance Observation Tool $^{65}$ can be used during simulations to guide debriefing around observable team behaviors related to patient safety. The role of team debriefing extends beyond the simulation settings, with recent studies reporting the use and benefits of clinical debriefings in real settings after actual clinical situations as a valuable tool to promote patient safety, teamwork, collaboration, and mutual support. $^{66-68}$

Although scarce literature exists on the impact of team training on patient outcomes, a previous medical team training program implemented across 108 Veteran Health Administration facilities, showed that interprofessional team training among surgical teams, using the principles of Crew Resource Management, was associated with lower surgical mortality after 1 year of the program implementation. ${ }^{69}$

Summarized practical advice toward developing highfunctioning teams can be gleaned from lessons learned, shown in Table 1 and displayed graphically in Figure 2. Furthermore, for each approach adopted, it is critical that team development interventions be evidence-based to result in measurable improvement in teamwork.

Implementing changes to the OR to ICU handover process and sustaining those changes is not an easy task. The authors have experienced firsthand several barriers to the implementation of a redesigned handover. In academic institutions, trainees rotate periodically through different units throughout the health system, which results in a continuous turnover of key members of the team. Training on a process that is specific to a single unit (in this case the CVICU) will therefore have to be repeated constantly until there is significant institutional culture change, which may take years. Additionally, significant differences in buyin may exist among staff in different units, even when both units are equally equipped to care for patients postcardiac 


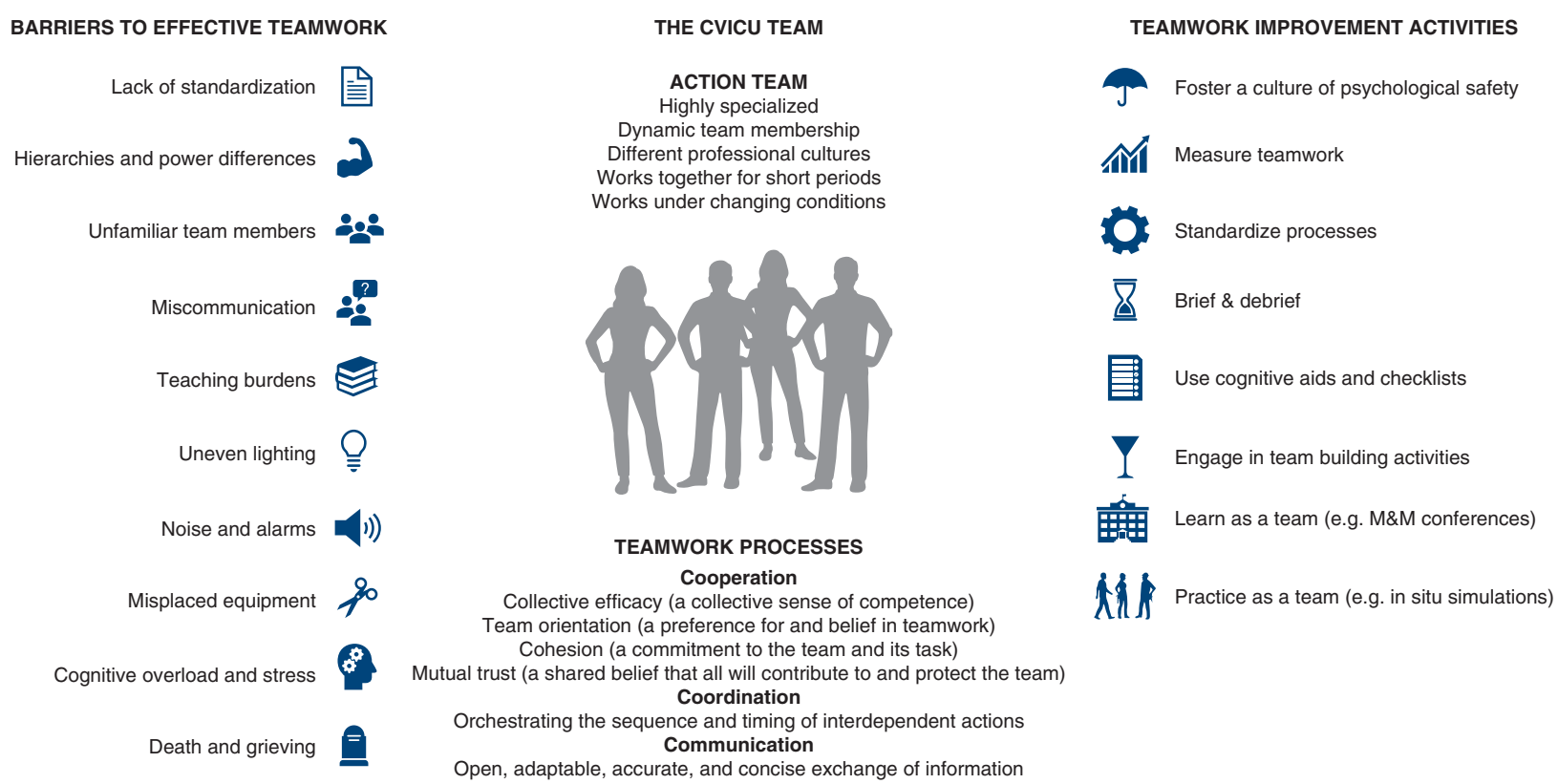

FIGURE 2. Cooperation, coordination, and communication underlie high-performing health care teams. On the left-hand side of this figure, specific individual, environmental, institutional, and cultural barriers to effective teamwork are outlined. Details describing the nature of the CVICU team and required teamwork processes are displayed in the middle column. The right-hand side of this figure lists specific activities suggested to improve CVICU teamwork, based on the author's experiences and evidence in the literature (A.B.). ${ }^{35} C V I C U$, Cardiovascular intensive care unit; $M \& M$, Morbidity and mortality.

surgery. Therefore, the handover experiences may look different in each of these units. Differences in the fidelity of the implementation may also exist between day-shift and night-shift ICU staff (day-shift staff have more chances of being actively involved in the process redesign, which may influence their engagement).

\section{FAILURE TO RESCUE}

FTR, defined as "mortality among patients with a major postoperative complication" is an important factor underlying postoperative mortality. ${ }^{75}$ Furthermore, FTR rates have been demonstrated to be a significant source of interhospital variability ${ }^{76}$ and an important determiner of total mortality of surgery. ${ }^{77,78}$ In fact, FTR rates vary more than the risk-adjusted rate of complications, ${ }^{79-81}$ with FTR rates varying between hospitals by as much as a factor of $6 .{ }^{82}$ In cardiac surgery in particular, a recent observational study identified high interhospital variability among successful rescues, despite similar complication rates. ${ }^{83}$ Thus, although efforts to reduce the rate of complications are certainly important, reducing mortality may depend more on what happens after complications occur.

A number of studies have investigated the relation between FTR rates and hospital characteristics, such as number of ICU beds, nurse-to-patient ratio, hospital technology, and hospital procedural volume, ${ }^{78,82,84}$ but these characteristics account for only approximately one-third of the variation in FTR rates. Low FTR rates seem to depend strongly on the characteristics and experience of the ICU teams, but in ways that are not well understood or quantified.

Successful rescue requires both early recognition of serious complications and timely and effective management of them once they have occurred. In particular, quickly recognizing the possibility of a complication requires awareness of many factors, including current physiological data on the patient and information about ongoing treatment as well as historical information. Recognition depends on the ICU team having a high "index of suspicion" when interpreting rapidly changing or conflicting data. Once the possibility of a specific complication has been recognized, timely and effective management requires coordination of the activities of the ICU team. The response to a suspected complication may itself be complex, involving a number of individuals coordinating their activities and possibly requiring the ICU team to get additional information to sharpen the diagnosis and then modify their activities accordingly. Such responses must take into account not just one suspected complication, but the team understanding of the entire situation. ${ }^{85,86}$

Recently, the Society of Thoracic Surgeons' Adult Cardiac Surgery National Database was queried to provide insight into the role of FTR in coronary artery bypass graft surgery operations ${ }^{86}$ : Major limitations of the Society of Thoracic Surgeons' Adult Cardiac Surgery National Database are that data are collected in a voluntary manner, the timing of complications is not collected, and suboptimal 
data quality may distort analysis and conclusions. With these caveats in mind, the authors described an interesting paradox: Centers with the lowest complication rate had the highest FTR (FTR observed/expected ratio 1.14) whereas those with the highest complication rates had the lowest FTR (FTR observed/expected ratio 0.91).

The authors propose as an explanation of this paradoxical finding that centers with high complication rates may have more experience treating complications and be likely to salvage (rescue) patients who have these complications. An alternative explanation is that the authors' arbitrary choice of composite complications, the voluntary nature of the Society of Thoracic Surgeons database, and their less granular choice of terciles versus quintiles may have led them to the wrong conclusion.

Regardless of the methods and the subset of procedures studied, FTR is regarded as a reliable index of the prompt recognition and effective treatment of postoperative complications, reflecting perhaps a more effective "system of care." Therefore, patient safety may be improved by addressing the factors that influence escalation of care and FTR, especially in the high-consequence CVICU setting. Efforts to improve human factors may in fact be an undervalued approach to decreasing FTR. The importance of teamwork, leadership and, more in general, of healthy interprofessional relationships in the ICU setting cannot be underestimated. In addition, a recent literature review also raised concerns regarding hierarchy and overconfidence factors and points to sobering issues surrounding the structure of the surgical and critical care teams and institutional culture. ${ }^{87}$ These factors may be the result of a poorly managed system, leading to a culture that does not support patient safety and reporting of adverse events. An improved culture of safety has been linked with fewer incidents of errors in patient safety and better outcomes. ${ }^{88}$ However, it is not currently established whether FTR rates are influenced by human factors, because the literature has mostly explored the role of human factors in the OR but has neglected to study their impact on ICU or ward-based care. This concept should be the focus of future research.

\section{Conflict of Interest Statement}

The authors reported no conflicts of interest.

The Journal policy requires editors and reviewers to disclose conflicts of interest and to decline handling or reviewing manuscripts for which they may have a conflict of interest. The editors and reviewers of this article have no conflicts of interest.

\section{References}

1. Wahr JA, Prager RL, Abernathy III JH, Martinez EA, Salas E, Seifert PC, et al. Patient safety in the cardiac operating room: human factors and teamwork: a scientific statement from the American Heart Association. Circulation. 2013;128: 1139-69.
2. Salas E, Cooke NJ, Rosen MA. On teams, teamwork, and team performance: discoveries and developments. Hum Factors J Hum Factors Ergon Soc. 2008;50: $540-7$.

3. Suliburk JW, Buck QM, Pirko CJ, Massarweh NN, Barshes NR, Singh H, et al. Analysis of human performance deficiencies associated with surgical adverse events. JAMA Netw Open. 2019;2:e198067.

4. Salas E, Sims D, Burke CS. Is there a "big five" in teamwork? Small Gr Res. 2005;36:555-99.

5. Westli HK, Johnsen BH, Eid J, Rasten I, Brattebø G. Teamwork skills, shared mental models, and performance in simulated trauma teams: an independent group design. Scand J Trauma Resusc Emerg Med. 2010;18:1-8.

6. Brown EKH, Harder KA, Apostolidou I, Wahr JA, Shoo DC, Farivar RS, et al. Identifying variability in mental models within and between disciplines caring for the cardiac surgical patient. Anesth Analg. 2017;125:29-37.

7. Rouse WB, Cannon-Bowers JA, Salas E. The role of mental models in team performance in complex systems. IEEE Trans Syst Man Cybern Syst. 1992;22: 1296-308.

8. Alsabri M, Boudi Z, Lauque D, Dias RD, Whelan J, Östlundh L, et al. Impact of teamwork and communication training interventions on safety culture and patient safety in emergency departments. J Patient Saf. September 7, 2020 [Epub ahead of print].

9. Donovan AL, Aldrich JM, Gross AK, Barchas DM, Thornton KC, SchellChaple HM, et al. Interprofessional care and teamwork in the ICU. Crit Care Med. 2018;46:980-90.

10. Havyer RDA, Wingo MT, Comfere NI, Nelson DR, Halvorsen AJ, McDonald FS, et al. Teamwork assessment in internal medicine: a systematic review of validity evidence and outcomes. J Gen Intern Med. 2014;29:894-910.

11. Shrader S, Kern D, Zoller J, Blue A. Interprofessional teamwork skills as predictors of clinical outcomes in a simulated healthcare setting. J Allied Health. 2013; 42:1E-6E.

12. Robertson JM, Dias RD, Yule S, Smink DS. Operating room team training with simulation: a systematic review. J Laparoendosc Adv Surg Tech. 2017;27: 475-80.

13. Mazzocco K, Petitti DB, Fong KT, Bonacum D, Brookey J, Grahma S, et al. Surgical team behaviors and patient outcomes. Am J Surg. 2009;197:678-85.

14. Makary MA, Sexton JB, Freischlag JA, Holzmueller CG, Millman EA, Rowen L, et al. Operating room teamwork among physicians and nurses: teamwork in the eye of the beholder. J Am Coll Surg. 2006;202:746-52.

15. Weller J, Boyd M, Cumin D. Teams, tribes and patient safety: overcoming barriers to effective teamwork in healthcare. Postgrad Med J. 2014;90:149-54.

16. Tsuburaya A, Soma T, Yoshikawa T, Cho H, Miki T, Uramatsu M, et al. Introduction of the non-technical skills for surgeons (NOTSS) system in a Japanese Cancer Center. Surg Today. 2016;46:1451-5.

17. Scott J, Revera Morales D, Mcritchie A, Riviello R, Smink D, Yule S. Non-technical skills and health care provision in low- and middle-income countries: a systematic review. Med Educ. 2016;50:441-55.

18. Pradarelli JC, Gupta A, Lipsitz S, Smink DS, Yule S. Evaluating the nontechnical skills for surgeons assessment tool adapted to the US surgical context. J Am Coll Surg. 2019;229:S242.

19. Scott DJ, Dunnington GL. The new ACS/APDS skills curriculum: moving the learning curve out of the operating room. J Gastrointest Surg. 2008;12: 213-21.

20. Kasaoka S. Evolved role of the cardiovascular intensive care unit (CICU). $J$ Intensive Care. 2017;5:1-5.

21. Janssens S, Simon R, Beckmann M, Marshall S. Shared leadership in healthcare action teams: a systematic review. J Patient Saf. June 4, 2018 [Epub ahead of print].

22. Edmondson AC. Speaking up in the operating room: how team leaders promote learning in interdisciplinary action teams. J Manag Stud. 2003;40:1419-52.

23. Dual training in adult cardiothoracic anesthesia|Society of Cardiovascular Anesthesiologists. Available at: https://www.scahq.org/dual-training-in-adultcardiothoracic-anesthesia/. Accessed February 5, 2021.

24. Stamou SC, Camp SL, Stiegel RM, Reames MK, Skipper E, Watts LT, et al. Quality improvement program decreases mortality after cardiac surgery. $J$ Thorac Cardiovasc Surg. 2008;136:6-11.

25. Katz JN, Minder M, Olenchock B, Price S, Goldfarb M, Washam JB, et al. The genesis, maturation, and future of critical care cardiology. J Am Coll Cardiol. 2016;68:67-79.

26. Au SS, Roze des Ordons AL, Parsons Leigh J, Soo A, Guienguere S, Bagshaw SM, et al. A multicenter observational study of family participation in ICU rounds. Crit Care Med. 2018;46:1255-62. 
27. Azoulay É, Timsit JF, Sprung CL, Soares M, Rusinová K, Lafbrie A, et al. Prevalence and factors of intensive care unit conflicts: the conflicus study. Am J Respir Crit Care Med. 2009;180:853-60.

28. Ervin JN, Kahn JM, Cohen TR, Weingart LR. Teamwork in the intensive care unit. Am Psychol. 2018;73:468-77.

29. Bartley J, Streifel AJ. Design of the environment of care for safety of patients and personnel: does form follow function or vice versa in the intensive care unit? Crit Care Med. 2010;38:S388-98.

30. Piquette D, Reeves S, LeBlanc VR. Stressful intensive care unit medical crises: how individual responses impact on team performance. Crit Care Med. 2009;37: 1251-5.

31. Segall N, Bonifacio AS, Schroeder RA, Barbeito A, Rogers D, Thornlow DK, et al. Can we make postoperative patient handovers safer? A systematic review of the literature. Anesth Analg. 2012;115:102-15.

32. Catchpole KR, De Leval MR, Mcewan A, Pigott N, Elliot MJ, McQuillan A, et al. Patient handover from surgery to intensive care: using formula 1 pit-stop and aviation models to improve safety and quality. Paediatr Anaesth. 2007;17:470-8.

33. Kowitlawakul Y, Leong BSH, Lua A, Aroos R, Wong JJ, Koh N, et al. Observation of handover process in an intensive care unit (ICU): barriers and quality improvement strategy. Int J Qual Health Care. 2015;27:99-104.

34. Gawande AA, Zinner MJ, Studdert DM, Brennan TA. Analysis of errors reported by surgeons at three teaching hospitals. Surgery. 2003;133:614-21.

35. Segall N, Bonifacio AS, Barbeito A, Schroder RA, Perfect SR, Wright MC, et al. Operating room-to-ICU patient handovers: a multidisciplinary human-centered design approach. Jt Comm J Qual Patient Saf. 2016;42:400-9. AP1-AP5.

36. Institute of Medicine. In: Kohn LT, Corrigan JM, Donaldson MS, eds. To Err Is Human: Building a Safer Health System. 71. Washington, DC: The National Academies Press; 2000.

37. Baggs JG, Schmitt MH, Mushlin AI, Mitchell PH, Eldredge DH, Oakes D, et al. Association between nurse-physician collaboration and patient outcomes in three intensive care units. Crit Care Med. 1999;27:1991-8.

38. Knaus WA, Draper EA, Wagner DP, Zimmerman JE. An evaluation of outcome from intensive care in major medical centers. Ann Intern Med. 1986;104:410-8.

39. Shortell SM, Zimmerman JE, Rousseau DM, Gillies RR, Wagner DP, Draper EA, et al. The performance of intensive care units: does good management make a difference? Med Care. 1994;32:508-25.

40. Barbeito A, Agarwala AV, Lorinc A. Handovers in perioperative care. Anesthesiol Clin. 2018:36:87-98.

41. Thomas EJ. Improving teamwork in healthcare: current approaches and the path forward. Postgrad Med J. 2012:88:245-8.

42. Rosen MA, Schiebel N, Salas E, Wu TS, Silvestri S, King HB. Improving patient safety through teamwork and team training. In: Salas E, Frush K, eds. How can team performance be measured, assessed, and diagnosed? Oxford, UK: Oxford University Press; 2012:59-79.

43. Jeffcott SA, Mackenzie CF. Measuring team performance in healthcare: review of research and implications for patient safety. J Crit Care. 2008;23:188-96.

44. Rosen MA, Dietz AS, Yang T, Priebe CE, Pronovost PJ. An integrative framework for sensor-based measurement of teamwork in healthcare. JAm Med Inform Assoc. 2014;22:11-8.

45. Zenati MA, Kennedy-Metz L, Dias RD. Cognitive engineering to improve patient safety and outcomes in cardiothoracic surgery. Semin Thorac Cardiovasc Surg. 2019;32:1-7.

46. Wadhera RK, Parker SH, Burkhart HM, Greason KL, Neal JR, Levenick KM, et al. Is the "sterile cockpit" concept applicable to cardiovascular surgery critical intervals or critical events? The impact of protocol-driven communication during cardiopulmonary bypass. J Thorac Cardiovasc Surg. 2010;139:312-9.

47. Foster S, Manser T. The effects of patient handoff characteristics on subsequent care: a systematic review and areas for future research. Acad Med. 2012;87: 1105-24.

48. Payne CE, Stein JM, Leong T, Dressler DD. Avoiding handover fumbles: a controlled trial of a structured handover tool versus traditional handover methods. BMJ Qual Saf. 2012;21:925-32.

49. Joy BF, Elliott E, Hardy C, Sullivan C, Backer CL, Kane JM. Standardized multidisciplinary protocol improves handover of cardiac surgery patients to the intensive care unit. Pediatr Crit Care Med. 2011;12:304-8.

50. Gleicher Y, Mosko JD, McGhee I. Improving cardiac operating room to intensive care unit handover using a standardised handover process. BMJ Open Qual. 2017;6:e000076.

51. Edmondson AC. Learning from mistakes is easier said than done: group and organizational influences on the detection and correction of human error. $J$ Appl Behav Sci. 2004;40:66-90.
52. Ridley CH, Al-Hammadi N, Maniar HS, Abdallah AB, Steinberg A, Bollini ML, et al. Building a collaborative culture: focus on psychological safety and error reporting. Ann Thorac Surg. 2021;111:683-9.

53. Eppich W, Howard V, Vozenilek J, Curran I. Simulation-based team training in healthcare. Simul Healthc J Soc Simul Healthc. 2011;6:S14-9.

54. Nunink L, Welsh AM, Abbey M, Buschel C. In situ simulation-based team training for post-cardiac surgical emergency chest reopen in the intensive care unit. Anaesth Intensive Care. 2009;37:74-8.

55. Figueroa M, Sepanski R, Goldberg S, Shah S. Improving teamwork, confidence, and collaboration among members of a pediatric cardiovascular intensive care unit multidisciplinary team using simulation-based team training. Pediatr Cardiol. 2013;34:612-9.

56. Sandahl C, Gustafsson H, Wallin CJ, Meurling L, Øvretveit J, Brommels M, et al Simulation team training for improved teamwork in an intensive care unit. Int $J$ Health Care Qual Assur. 2013;26:174-88.

57. Dunning J, Nandi J, Ariffin S, Jerstice J, Danitsch D, Levine A. The cardiac surgery advanced life support course (CALS): delivering significant improvements in emergency cardiothoracic care. Ann Thorac Surg. 2006;81:1767-72.

58. Daglius Dias R, Scalabrini Neto A. Stress levels during emergency care: a comparison between reality and simulated scenarios. J Crit Care. 2016;33: $8-13$.

59. Subbe CP, Kellett J, Barach P, Chaloner C, Cleaver H, Cooksley T, et al Crisis checklists for in-hospital emergencies: expert consensus, simulation testing and recommendations for a template determined by a multiinstitutional and multi-disciplinary learning collaborative. BMC Health Serv Res. 2017;17.

60. Sanko JS, McKay M. Participation in a system-thinking simulation experience changes adverse event reporting. Simul Healthc. 2020;15:167-71.

61. Davis WA, Jones S, Crowell-Kuhnberg AM, O'Keeffe D, Boyle KM, Klainer SB et al. Operative team communication during simulated emergencies: too busy to respond? Surgery. 2017;161:1348-56.

62. Agency for Healthcare Research and Quality. Using Simulation in TeamSTEPPS training: classroom slides. Available at: https://www.ahrq.gov/teamstepps/ simulation/simulationslides/simslides.html. Accessed December 15, 2020.

63. Cooper S, Cant R, Connell C, Sims L, Porter JE, Symmons M, et al. Measuring teamwork performance: validity testing of the Team Emergency Assessment Measure (TEAM) with clinical resuscitation teams. Resuscitation. 2016;101: 97-101.

64. Yule S, Flin R, Maran N, Rowley D, Youngson G, Paterson-Brown S. Surgeons non-technical skills in the operating room: reliability testing of the NOTSS behavior rating system. World J Surg. 2008;32:548-56.

65. Agency for Healthcare Research and Quality. Team performance observation tool. Available at: https://www.ahrq.gov/teamstepps/instructor/reference/tmpot html. Accessed December 15, 2020.

66. Servotte J-C, Bram Welch-Horan T, Mullan P, Piazza J, Ghuysen A, Szyld D. Development and implementation of an end-of-shift clinical debriefing method for emergency departments during COVID-19. Adv Simul (Lond). 2020;5:32.

67. Toews AJ, Martin DE, Chernomas WM. Clinical debriefing: a concept analysis. $J$ Clin Nurs. January 12, 2021 [Epub ahead of print].

68. Arriaga AF, Szyld D, Pian-Smith MCM. Real-time debriefing after critical events: exploring the gap between principle and reality. Anesthesiol Clin 2020:38:801-20

69. Neily J, Mills PD, Young-xu Y, Carney BT, West P, Berger DH, et al. Association between implementation of a medical team training program and surgical mortality. JAMA. 2010;304:1693-700.

70. Arriaga AF, Bader AM, Wong JM, Lipsitz SR, Berry WR, Ziewacz JE, et al Simulation-based trial of surgical-crisis checklists. N Engl J Med. 2013;368: 246-53.

71. Rasmussen MB, Tolsgaard MG, Dieckmann P, Østergaard D, White J, Plenge P, et al. Social ties influence teamwork when managing clinical emergencies. $B M C$ Med Educ. 2020;20:1-7.

72. Eddy K, Jordan Z, Stephenson M. Health professionals' experience of teamwork education in acute hospital settings: a systematic review of qualitative literature. JBI Database Syst Rev Implement Rep. 2016;14:96-137.

73. Lutgendorf MA, Spalding C, Drake E, Spence D, Heaton JO, Morocco KV Multidisciplinary in situ simulation-based training as a postpartum hemorrhage quality improvement project. Mil Med. 2017;182:e1762-6.

74. Steinemann S, Berg B, Skinner A, DiTulio A, Anzelon K, Terada K, et al. In situ, multidisciplinary, simulation-based teamwork training improves early trauma care. J Surg Educ. 2011;68:472-7. 
75. Silber JH, Williams SV, Krakauer H, Schwartz JS. Hospital and patient characteristics associated with death after surgery: a study of adverse occurrence and failure to rescue. Med Care. 1992;30:615-27.

76. Ghaferi AA, Birkmeyer JD, Dimick JB. Complications, failure to rescue, and mortality with major inpatient surgery in Medicare patients. Ann Surg. 2009; 250:1029-34.

77. Reddy HG, Shih T, Englesbe MJ, Shannon FL, Theurer PF, Herbert MA, et al. Analyzing "failure to rescue": is this an opportunity for outcome improvement in cardiac surgery? Ann Thorac Surg. 2013;95:1976-81.

78. Sheetz KH, Dimick JB, Ghaferi AA. Impact of hospital characteristics on failure to rescue following major surgery. Ann Surg. 2016;263:692-7.

79. Ghaferi AA, Birkmeyer JD, Dimick JB. Variation in hospital mortality associated with inpatient surgery. N Engl J Med. 2009;361:1368-75.

80. Glance LG, Dick AW, Meredith JW, Mukamel DB. Variation in hospital complication rates and failure-to-rescue for trauma patients. Ann Surg. 2011;253:811-6.

81. Pasquali SK, He X, Jacobs JP, Jacobs ML, O’Brien SM, Gaynor JW. Evaluation of failure to rescue as a quality metric in pediatric heart surgery: an analysis of the STS congenital heart surgery database. Ann Thorac Surg. 2012;94:573-80.

82. Ghaferi AA, Osborne NH, Birkmeyer JD, Dimick JB. Hospital characteristics associated with failure to rescue from complications after pancreatectomy. $J$ Am Coll Surg. 2010;211:325-30.
83. Likosky DS, Strobel RJ, Wu X, Kramer RS, Hamman BL, Brevig JK, et al. Interhospital failure to rescue after coronary artery bypass grafting. J Thorac Cardiovasc Surg. 2021 [In press].

84. Gonzalez AA, Dimick JB, Birkmeyer JD, Ghaferi AA. Understanding the volume-outcome effect in cardiovascular surgery: the role of failure to rescue. JAMA Surg. 2014;149:119-23.

85. Silber JH, Kaestner R, Even-Shoshan O, Wang Y, Bressler LJ. Aggressive treatment style and surgical outcomes. Health Serv Res. 2010;45(6 Part 2): 1872-92.

86. Edwards FH, Ferraris VA, Kurlansky PA, Lobdell KW, He X, O'Brien SM, et al. Failure to rescue rates after coronary artery bypass grafting: an analysis from the Society of Thoracic Surgeons Adult Cardiac Surgery database. Ann Thorac Surg. 2016;102:458-64.

87. Johnston MJ, Arora S, King D, Bouras G, Almoudaris AM, Davis R, et al. A systematic review to identify the factors that affect failure to rescue and escalation of care in surgery. Surgery. 2015;157:752-63.

88. Singer S, Lin S, Falwell A, Gaba D, Baker L. Relationship of safety climate and safety performance in hospitals. Health Serv Res. 2009;44(2P1):399-421.

Key Words: cognitive workload, coordination, cooperation, patient safety, teamwork 